\title{
Traffic Management in 5G Mobile Networks: Selfish Users and Fair Network
}

\author{
Menglan Jiang and Toktam Mahmoodi \\ Department of Informatics, King's College London, UK \\ menglan.jiang@kcl.ac.uk; toktam.mahmoodi@kcl.ac.uk
}

\begin{abstract}
With increasing in heterogeneity of the mobile and wireless networks, including the use of licensed and unlicensed spectrum, and diversity in types of User Equipment (UEs), managing how traffic flows through network while maintaining high level of users' quality of experience is crucial. In this paper, we present a novel traffic management mechanism that maintains the users' quality of experience as well as guaranteeing fairness among users. This traffic management has two separate elements, one that is located at the UE and one that is located at the cloud-based network controller. While each UE maximizes their utility function, which is modelled based on the QoS parameters, selfishly, at the network side, the attempt is to maximize fairness among all users' flows.
\end{abstract}

Keywords: 5G mobile; QoS; Fairness; traffic management; LTE; WiFi; SDN; central controller.

\section{Introduction}

The next generation of mobile networks, a.k.a. 5G, will be deployed with dense small cells of different technologies including LTE femtocells and picocells, WiFi access points, and novel radios, such as millimeter wave. Faced with an ever larger portfolio of applications to serve and with a corresponding number of requirements to satisfy, it is commonly recognized that $5 \mathrm{G}$ need to consider various requirements of different application domains and industry sectors.

To address the above, there has been number of initiatives for the design of new mobile network architecture. One of the avenues for the $5 G$ architecture design is the fully decoupled architecture. Decoupling of uplink and downlink has been well studied over the past few years [1] and its pros and cons are discussed in the community. Decoupling of the control and data plane is another well-investigated topic that is mostly studied within the context of Software-defined Networking (SDN) [2], [3]. Virtualisation and cloudification of the mobile networking functionalities is another element of $5 G$ network that is enabled in such a decoupled architecture [4].

On the other hand and with the ever increasing data traffic in mobile networks, traffic management and maintaining Quality of Service (QoS) is more than ever challenging. According to Cisco Visual Networking Index, global mobile data traffic reached the 2.5 exabytes per month at the end of 2014, and this figure will surpass 24.3 exabytes by 2019 [5]. Hence, more efficient traffic managements are needed that can deal with the backhaul congestion, and guarantee the QoS for users. In the SDN-based 5 G network, it has 
Menglan Jiang and Toktam Mahmoodi; Traffic Management in 5G Mobile Networks: Selfish Users and Fair Network, Transactions on Networks and Communications, Volume 4 No. 1, February (2016); pp: 1-15

been shown that centralized traffic management mechanism can provide guaranteed QoS and more efficient traffic management [6].

To this end, we discussed a device-controlled mechanism in our previous work [7], where all decisions are made at the User Equipment (UE). Such device-controlled decision making mainly focuses on the users' QoS requirements and is a fully "selfish" decision. We designed the algorithm for selecting radio access at the UE with a reinforcement learning process that takes Received Signal Strength (RSS) and battery status of the UE into consideration. Here, we extend our previously designed device-controlled traffic management to address the issue of fairness, i.e. while users maximize their own interest, network maintains fairness among users.

Therefore, we define a traffic management mechanism based on "selfish" users and "fair" network, where the network side is a cloud-based central controller. We use Jain's fairness index to quantify fairness [8], and simulated annealing as a heuristic to solve the optimization problem at the controller side. The Jain's index has been well-used for quantifying fairness in communication networks [9]. At the UE side, the problem of long-term QoS maximization is formulated as a Q-learning problem. The contributions of this paper are threefold:

- We propose a new QoS-based traffic management mechanism which can maximizes QoS utility of each UEs while implementing fairness maximization for the network system. In this paper, we call this scheme QoS and Fairness maximization (QFM). We use Jain's fairness to define fairness among UEs and assume such fairness in maintained at the "central controller". Hence, the final decisions of network selection will be made by both UE and central controller together in order to maximize QoS utility of the UEs as well as Jain's fairness index between all traffic flows.

- We maximize fairness levels of the system with QoS values constraints. It can be implemented by our system model which combines device-controlled mechanism and cloud central controller together. Device-controlled mechanism has been explained in our previous work which is a fully distributed mechanism used to consider UEs' location information and their QoS requirements. In this paper, we add a cloud central controller on top of the whole system and devise an optimization approach to ensure traffic resource has been effectively managed based on our QFM mechanism.

- When backhaul congestion has been taken into account, we can maximize UEs' QoS values by using channels with less congestion. Backhaul congestion tends to decrease the overall system performance and generate unfairness issues among UEs. In this paper, instead of measuring oneway packet delay to detect congested transport backhaul link in LTE networks which has been proposed in existing literature [10], we propose a novel approach using central controller on top of the system to provide information of backhaul links to UEs in the future networks.

The remainder of this paper is organized as follows. Section II briefly reviews the state of art for cloudbased central controller and fairness in traffic management. After elaborating our system model and fairness approach in Section III, our problem formulation and traffic management approach will be described in Section IV and V, respectively. Section VI presents simulation study and performance observations. Finally, highlights of this work and road ahead are discussed in Section VII. 


\section{Related Works}

In this section, we review the state of art in cloud-based control plane and also traffic management mechanisms in mobile networks. There is a large body of research on virtualization of mobile network functions and the design of different architecture for cloud-base control plane [10]. Using the SDN paradigm for decoupling data and control and managing network centrally has also been discussed in the $5 G$ literature. Examples of such work are the presented research in [2], [3] and [4]. In [2], new SDN-based architecture for $5 \mathrm{G}$ is presented so as to reduce latency for mission critical applications. In [3], it has been shown how logically central controller can be placed in the LTE network architecture. The effect of such architecture design on signalling overhead and agility of control are also discussed in this paper. Furthermore, research work in [4] focuses on the design of access cloud in SDN-based 5G architecture.

On the other hand, the explosive growth of cloud-based applications for mobile devices, brought attention to the development of networking architectures and mechanisms to assist operators in managing traffic as dynamically as possible. Well-designed traffic management will allow network operators to draw maximum value from available capacity by managing network resources as efficiently as possible. Running traffic management at the UEs side allow such decision to be made where the required information are available in the most up-to-date and precise format (all measured at the UE and utilised at the UE). Hence, users can potentially achieve their desired QoS level that is either improving their received data rate, and communication latency or lowering their power consumption. In this regard, the device-centric network architecture has been listed as a solution to address users' stringent QoS requirements in [11]. In [12], automatic Access Network Selection (ANS) has been proposed in a device-controlled manner for better traffic management. Furthermore, various ANS mechanisms for enabling "always-best" connectivity are reviewed in [13], and it has been concluded that introduction of cognition and advanced learning capabilities, can act as a catalyst for improving the quality of ANS decisions. Based on the discussed literature here, we propose a dynamic traffic management mechanism within SDN-based 5G architecture, and with integrating learning capabilities based on the analytics of networks.

Cognition and learning capabilities have been introduced in different aspects of mobile networks including routing, resource management and dynamic channel selection [14], [15]. We use Q-learning in this paper that is a model-free reinforcement learning technique. Q-Learning and reinforcement learning are frequently used in the mobile and wireless networks. An online path selection algorithm based on Qlearning has also been proposed in [16] for minimizing the probability of burst loss in optical switching networks. In [17], fuzzy Q-learning algorithm is used to optimize call dropping rate for traffic steering.

The last topic we touch on, in our background section is consideration of fairness in mobile networks. Fairness has been well-studied in the context of scheduling and wireless recourse allocations, either on the wireless channel or over the end-to-end flow $[18,19]$. Similarly fairness has been studied in workload distribution in Datacenters [20]. To quantify fairness, various different fairness measures have been proposed in the literature. The Jain's fairness index [8], which was conceived to measure fairness in computer networks, is a very well used measure of fairness in both wired and wireless networks [21], thanks to its advantageous mathematical properties. Therefore, we also use Jain's index to measure fairness in this paper. 


\section{System Model}

\subsection{Model of the system-level architecture}

Different elements of our proposed QoS and fairness maximization (QFM) traffic management is detailed in this section, and depicted in Figure 1. The three main layers in this model are the UE layer, the wireless network layer (i.e. radio access and mobile core, e.g. EPC), and the cloud layer (i.e. central controller). These three layers are elaborated here and the flow chart of communication between these layers has been described in Fig 2.

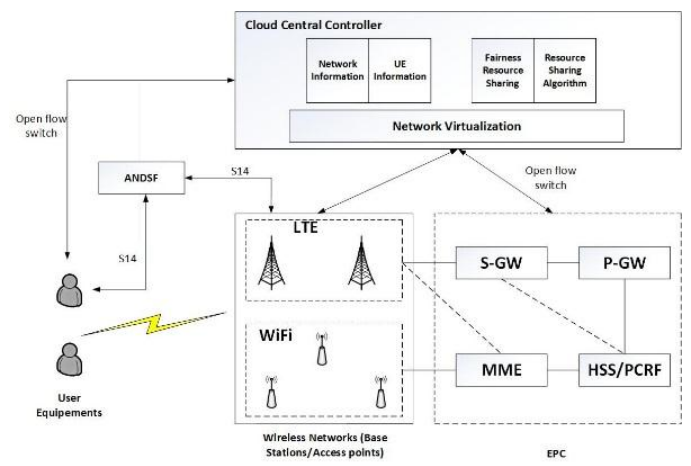

Figure 1. SDN-based System Architecture.

- UE Layer: In our model, UEs can communicate with the Access Network Discovery and Selection Function (ANDSF) server via the S14 interface [22]. We further assume ANDSF includes network analytics server and UE can acquire network analytics through S14. The analytics, we consider here, are performance of different RANs in terms of QoS level for the UEs previously connected to the RAN. This information can be collected from database Candidate Networks Information (CNI) which connects with ANDSF directly. The central controller can also communicate with UEs through open interfaces, i.e. the OpenVSwitch on the mobile device operating systems, so that controller's decision on fairness maximization can be communicated to the UEs.

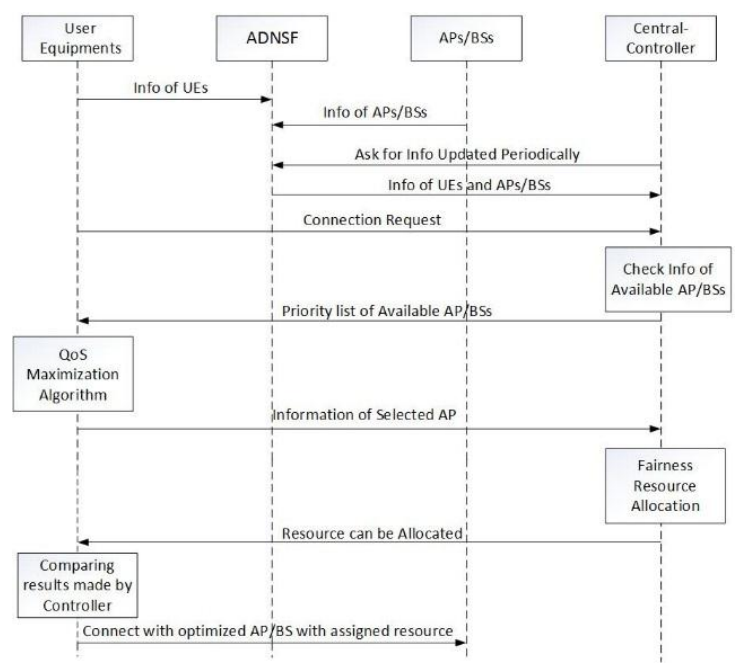

Figure 2. Communication Architecture 
- Wireless Network Layer: The wireless network layer comprises of the radio access and mobile core network that provide connectivity to UEs. We model the radio access network with four different Access Points (AP), consisting of LTE macro $(i=1)$, pico $(i=2)$ and femto $(i=3)$ as well as one WiFi access point $(i=4)$. The coverage area of these wireless access points is a circle with diameter $R_{i}$ meter, where $R=\{500 ; 300 ; 50 ; 100\}$ (values from [23]). For simplicity, both of the WiFi AP and cellular base stations are referred to as wireless AP from this point on. The core network consists of serving gateway (S-GW), packet data network gateway (P$\mathrm{GW}$ ), mobility management element (MME) and policy and charging rules function (PCRF) that have been used to implement connection, mobility and QoS management.

- Cloud Central Controller: Our cloud-based central controller implements the following rules:

(1) based on periodically updated information from UEs and APs, central controller can check if any of the AP is available. If only one AP is available for a given UE, controller will assign this AP to the UE; (2) if more than one AP is available for the UE, controller will run fairness maximization algorithm and provide the priority list of available APs to the access network selection at the UE.

\subsection{UE's battery Models}

The UE's battery consumption and how it will be affected by the application's throughput, is modeled here. We use the model described in [27] for the battery discharging rate, $\zeta$, during the lifetime of battery, $\mathrm{T}$, based on Equation (1).

$$
\zeta(T)=\frac{\pi^{2}}{3 \beta^{2}} e^{-\beta^{2} T}
$$

where $\beta$ is the value of chemical parameter and may vary from battery to battery in the range of $(0.4,1)$. It has been shown in [28] that by running three different applications concurrently on various smart phones, their battery lasts for two hours. Therefore, $\mathrm{T}$ is set to 120 minutes working time is the value we used for our simulations.

The energy consumption of the device, when connected to the $i$-th AP with distance $d$ is detailed in Equation (2).

$$
E_{i}=\zeta(T)+\eta d_{i}^{n}
$$

where $\eta$ and $n$ denote the battery consumption per distance unit, and the propagation loss coefficient, respectively [28].

\section{Problem Formulation}

In this section, we present details of the optimization problems that formulate our proposed QMF mechanism. We will describe two sets of problems, first those who describe the decision making of selfish users and then those that depict fairness maximization at the network. Throughout the problem formulation, we interchangeably use UE and user, assuming each user corresponds to a unique device.

\subsection{Selfish Users}

In our designed traffic management, UEs aim to maximize their QoE. We define QoE as a utility function based on received throughput and the battery consumption, as Equation (3).

$$
\Omega=\frac{\left[\sum_{i} C_{i j}\right]^{w_{1}}+\left[\sum_{i} E_{i j}\right]^{w_{2}}}{\sum_{i} C_{i j}+\sum_{i} E_{i j}}
$$


Menglan Jiang and Toktam Mahmoodi; Traffic Management in 5G Mobile Networks: Selfish Users and Fair Network, Transactions on Networks and Communications, Volume 4 No. 1, February (2016); pp: 1-15

where $C_{i j}$ and $E_{i j}$ represent values of received throughput, and consumed energy by user $\mathrm{j}=\{1,2, \ldots, \mathrm{U}\}$ as a result of connection to the $\mathrm{i}$-th $\mathrm{AP}, \mathrm{i}=\{1,2, \ldots, \mathrm{N}\}$. The first term in the numerator shows the total received throughput by user $\mathrm{j}$, in case this user is connected to multiple APs. Similarly the second term is the total energy consumption at the UE. The $w_{1}$ and $w_{2}$ are weight values, representing the significance of these two different criteria in the utility. We further assume that backhaul congestion affects UE's throughput. We model the backhaul link of each AP as a queue with exponentially distributed service time, $1 / \mu_{i}$. Assuming $\sigma_{i j}$ is the received throughput over the wireless channel (using Shannon equation), then the received throughput through the $\mathrm{i}$-th $\mathrm{AP}$ is $C_{i j}=\min \left\{\sigma_{i j}, \mu_{i}\right\}$.

To this end, the following optimization problem will be solved at each UE, i.e. $U E_{j}$.

$$
\text { (P1): Maximize } \Omega_{j}
$$

Subject to:

$$
\sum_{i} E_{i j} \leq P_{j} \quad i=\{1,2, \ldots N\}
$$

Where $P_{j}$ denotes the remaining battery at the j-th UE.

\subsection{Fair Network}

The cloud-based controller aims to maximize fairness among all connected UEs. As explained earlier, we use Jain's fairness index to quantify the achieved fairness among UEs as a result of our proposed traffic management. Jain's fairness index can be explained as:

$$
J(X)=\frac{\left(\sum_{j=1}^{U} x_{j}\right)^{2}}{U * \sum_{j=1}^{U} x_{j}^{2}}
$$

Where $x_{j}=\sum_{i} C_{i j}$, that is the total received throughput by $U E_{j}$.

Therefore, the optimization problem at cloud-based controller can be formulated as:

(P2): Maximize $J(X)$

Subject to:

$$
x_{j} \geq C_{j}^{\min } \quad \forall j \in\{1, \ldots, U\}
$$

Where, $C_{j}^{\text {min }}$ show the QoS requirement (in this case minimum required throughput) of user $\mathrm{j}$ depending on its application.

\section{Traffic Management Solutions}

We present different solutions for the proposed traffic management optimizations in section IV. First, we use Q-learning to solve the selfish user optimization problem (P1) and then we use simulated annealing as a metaheuristic to solve the fairness maximization problem (P2).

\subsection{Solutions to (P1) Using Q-learning}

In order to solve (P1) for QoS optimization, we use Q-learning. The main reason for using a learning based approach is the possibility of including historical data so as to make a decision that is optimal choice for longer period of time, and to potentially reduce the number of handovers. 
Q-learning is an incremental dynamic planning process, which can be used to determine the optimal strategy through step by step approach. Hence, we need to define time-varying states, actions and reward function for the process of selecting the AP. At each time $t, s(t)$ describes the state of a given AP, which will alter to $s(t+1)$ by executing action $a(t)$. The Q-value of this transition is defined as the expected value in Equation (5).

$$
Q^{(t)}(s, a)=E\left\{R_{t} \mid s=s(t), a=a(t)\right\} .
$$

The state $s(t)$, action $a(t)$ and reward value of $R(t)$ are,

- $s_{i}(t)$ : State of $A P_{i}$ at time $t$ is denoted by $s_{i}(t) \in S$ and represent receiving service through $A P_{i}$.

- $a_{i}(t)$ : Actions $a_{i}(t) \in A$ represent changing from one AP to another.

- $\quad R_{i}(t)$ : We define the "Reward Function" based on the value of $\Omega$ in Equation (3). Equations (8) and (9) describe the immediate reward, $r_{i}(t)$, and the weighted and aggregated reward function over time, $R_{i}(t)$.

$$
\begin{gathered}
r_{i}(t)=\left(\sum_{i} \Omega_{j}(t)-\sum_{i} \Omega_{j}(t-1)\right), \\
R_{i}(t)=\sum_{k=1}^{10} \gamma^{k} r_{i}(t-k),
\end{gathered}
$$

Where $k$ demonstrate number of historical records that are taken into account and $\gamma$ is the discount factor. In other words, $\gamma$ represents significance of the previously recorded reward values on $R_{i}(t)$. In the simulation study of this paper, we set $\gamma=0.995$ and the ten time stamps in Equation (9) similar to the described algorithm in [14].

Based on parameters described above, we can calculate Q-values by considering historical records, as follows:

$$
Q^{(t)}(s, a)=Q^{(t)}(s, a)+\alpha\left[R(t)+\gamma Q^{(t)}(s, a)-Q^{(t-1)}(s, a)\right]
$$

where $Q^{(t)}(s, a)$ is the current value of $Q$ for a given AP at time $t$, and $Q^{(t-1)}(s, a)$ is the historic value that was stored in the $\mathrm{CNI}$ and retrieved by the UE. Parameter $\alpha$ represents the learning rate, that is a value in the range of $(0,1)$, if $\alpha=0$, the $Q$ value is never updated. Summary of the $Q$-learning algorithm for solving (P1) is described in Algorithm 1.

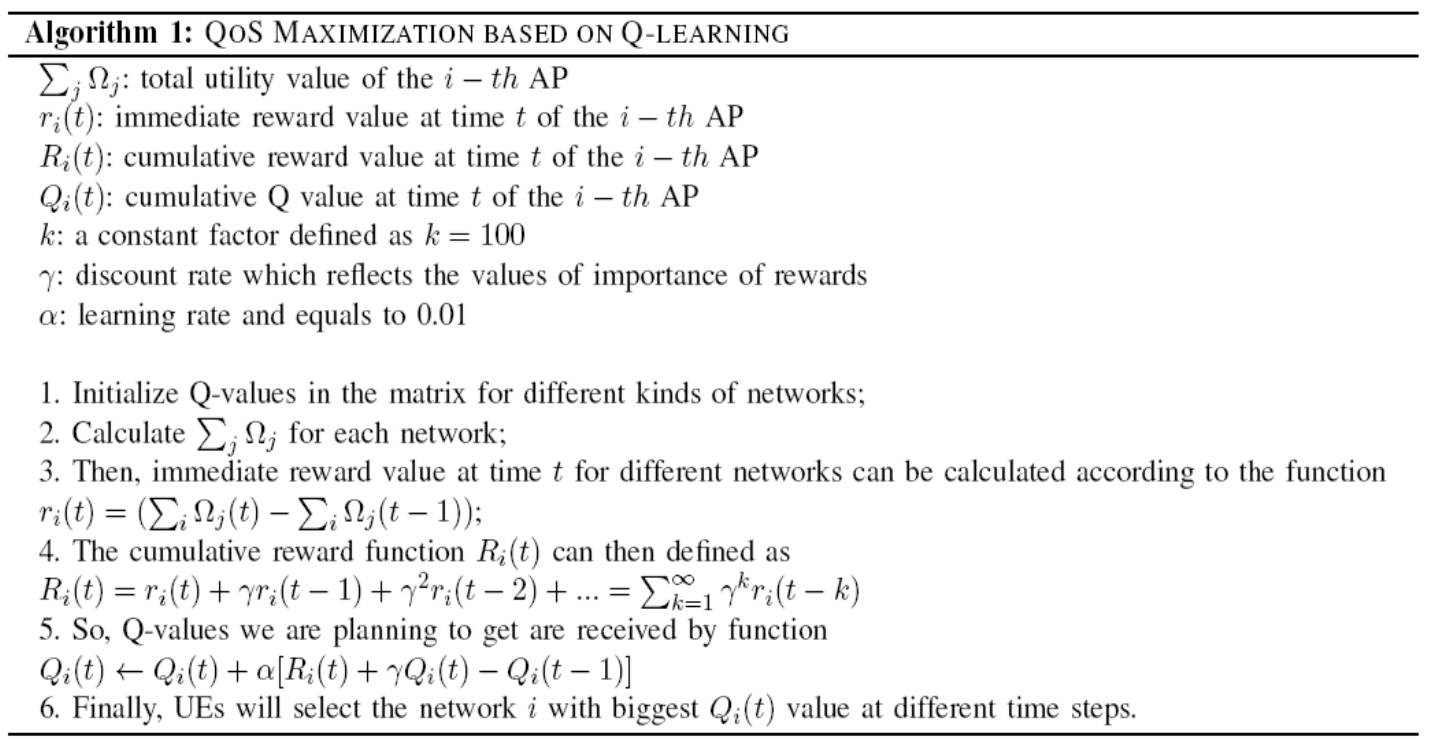




\subsection{Solution to (P2) using Simulated Annealing}

Simulated Annealing (SA) is a well-used heuristic for solving combinatorial problems. At each step of the SA algorithm, current solution will be replaced with a new solution given a certain probability. That probability depends on both difference between the current solution and randomly generated neighbor solution and also the temperature value T of the system [24].

In this section, we describe a solution for (P2) based on simulated annealing algorithm, which runs at the central controller. This algorithm, detailed in Algorithm 2, maximizes achieved fairness among the UEs. We are using Jain's fairness index as explained in IV-B to quantify fairness. Solving (P2), using the SA algorithm to consider maximizing $J(X)$. If the value of $J(X)$ for the neighbor AP is higher than the current one, the algorithm triggers a move to the neighboring AP. Otherwise, the algorithm choose an AP between the current AP and the neighbor AP according to a generated probability value. The random selection will allow solution to converge to global optimal point. The generated probability value for replacing current AP to the neighbor AP is based on $p=\frac{\Delta J(X)}{T}$, where $\Delta J(X)$ shows the difference of $\mathrm{J}(\mathrm{X})$ value between the current AP and the neighbor one.

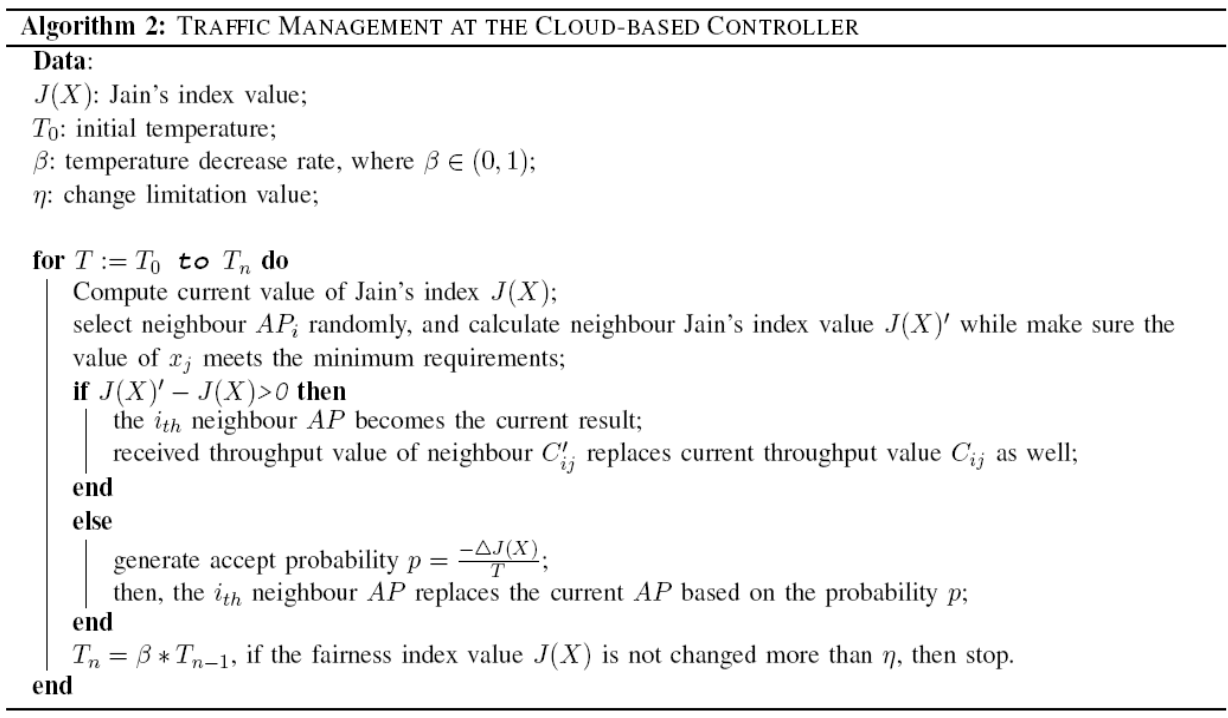

\section{Performance Evaluations}

In this section, we explain our simulation settings, discuss, and analyze the results.

\subsection{Simulation Parameters}

As mentioned earlier, we have modeled our system as an integrated wireless network that has four APs: one LTE macro cell, one pico cell and one femto cell, as well as one WiFi access point. We assume coverage areas of the three latter access points are included in the coverage area of the macro cell. The wireless channel is modeled with path loss (see Table I), and hence the RSS can be explained as $R S S_{i}=T P_{i}-$ $P L\left(d_{i}\right)$, where $T P_{i}$ denotes the transmit power of the $i$-th AP, $d_{i}$ is the distance between UE and the $i$-th AP and $P L\left(d_{i}\right)$ is the associated path loss value [25]. Detailed simulation parameters are described in Table 1. 
Table 1: Simulation Parameters

\begin{tabular}{|c|c|c|}
\hline Parameter & & Value \\
\hline \multirow{3}{*}{ Peak Data Rate } & LTE & $100 \mathrm{Mbps}$ \\
\cline { 2 - 3 } & WiFi & $11 \mathrm{Mbps}$ \\
\hline \multirow{4}{*}{ Tx Power } & LTE Macro & $46 \mathrm{dBm}$ \\
\cline { 2 - 3 } & LTE Pico & $23 \mathrm{dBm}$ \\
\cline { 2 - 3 } & LTE femto & $13 \mathrm{dBm}$ \\
\cline { 2 - 3 } & WiFi & $20 \mathrm{dBm}$ \\
\hline Noise Spectral Density & & $128.1+37.6 \mathrm{log}(\mathrm{d})$ \\
\hline \multirow{3}{*}{$\begin{array}{c}\text { Application } \\
\text { throughput }\end{array}$} & Video & $500-700 \mathrm{Kbps}$ \\
\cline { 2 - 3 } & Interactive & $300-600 \mathrm{Kbps}$ \\
\cline { 2 - 3 } & P2P & $700-1000 \mathrm{Kbps}$ \\
\cline { 2 - 3 } & E-service & $600-800 \mathrm{Kbps}$ \\
\hline Cell Coverage & LTE Macro & 500 meter \\
\cline { 2 - 3 } & LTE Pico & 300 meter \\
\cline { 2 - 3 } & LTE femto & 50 meter \\
\cline { 2 - 3 } & WiFi & 100 meter \\
\hline
\end{tabular}

\subsection{Simulation Scenarios}

- Scenario One: QoS-based traffic mechanism: In this scenario, we examine QoS-based RAN selection without considering history records. Weight values of different criteria have been set as 0.8 for received throughput and 0.2 for consumed energy by UEs, respectively. We assume decisions have been made by the UEs that can communicate with the ANDSF directly in order to receive information of candidate APs as described in 3.1. Therefore, UEs selects the AP that offers highest value of QoS utility, based on Equation (3).

- Scenario Two: learning-based traffic mechanism: In this scenario, we examine reinforcement learning based RAN selection. Compared with scenario one, we run Q-learning algorithm at UEs side by considering history Q-values of each available APs. This is based on solving P1 as explained in section 5.1. The main aim of using history values is to reduce the potential number of handovers by selecting the AP that has high performance over a period of time (and not only instantaneously).

- Scenario Three: QFM-based traffic mechanism: In this scenario, we examine our proposed QoS and Fairness maximization (QFM) based RAN selection. In scenario three we solve (P1) using Qlearning at the UE side and (P2) at the network controller side. ??? If there exists conflict between the results from the UE side and network controller side, the received throughput values by $U E_{j}$ should be checked simultaneously. If received throughput values that generated from the network controller side are in the field of application throughput described in Table 1, then the process of selecting APs is based on the results generated from the network controller side. Otherwise, the selected APs are based on the results generated from UE side.

\subsection{Result Analysis}

Our considered Key Performance Indicators (KPI) are: Users' throughput, UEs' battery consumption, number of handovers and Jain's fairness index. 


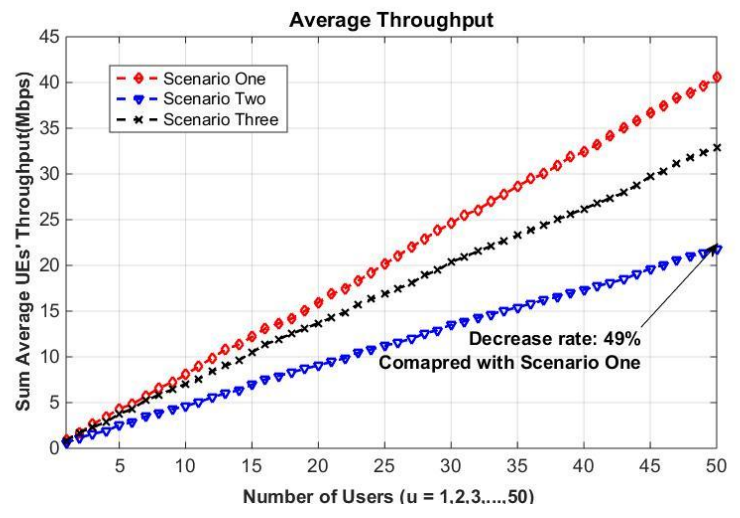

Figure 4. Sum throughput Vs. number of active users

Figure 4 indicates aggregated average received throughput for UEs in three different scenarios as we described above. Observing from this figure, it can be seen that throughput values in scenario one are the highest. That is because, the aim of QoS-based traffic mechanism in scenario one is maximizing QoS values based on Equation (3). Maximizing QoS values means maximizing the value of UEs received throughput. Since backhaul congestion are also considered in Scenario one, users were able to connect with an AP that provides higher throughput (and not only higher data rate over the wireless channel). Therefore, the value of sum average throughput UEs received in scenario one is higher than that in scenario two and three.

In scenario two, the sum average UEs' throughput is approximately $50 \%$ lower than those of scenario one. That is because, the main aim of learning-based traffic mechanism in this scenario is reducing the potential number of handovers without focusing on improving UEs received throughput. Therefore, the value of throughput in this scenario has decreased dramatically and from this figure not all UEs receive their required throughput values.

In scenario three, it can be explicit shown that the sum average UEs' throughput is lower than that in scenario one but higher than that in scenario two. That is because, the aim of QFM-based traffic mechanism is improving fairness allocation for all UEs which is restricted by achieving minimum UEs required throughput. Therefore, the value of sum throughput should be increased compared with scenario two but still reduced compared with that in scenario one which is mainly focus on maximized throughput values.

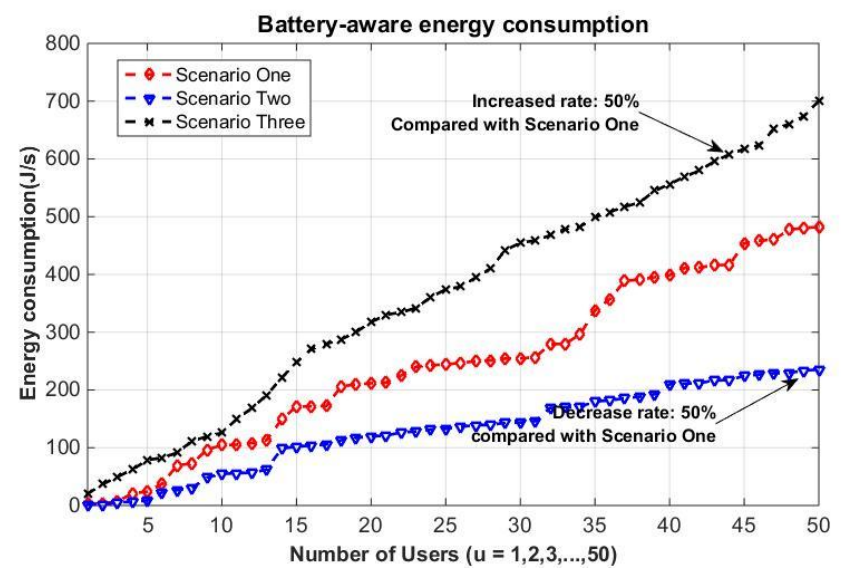

Figure 6. UEs' average battery consumptions 
Figure 6 shows battery consumption of the UEs in three different scenarios. It can be seen that values of battery consumption in scenario two are the lowest, and those values in scenario three are the highest. Because in scenario two, Q-learning algorithm has been implemented at UEs side. Cumulative reward values can help UEs to learn from history experience of candidate networks and can help them to perform best actions at each time steps. The aim of using Q-learning is reducing number of handovers for all UEs to help them maintain their ongoing communications for longer period of time. Therefore, the value of battery consumption should be reduced in scenario two and it is lower compared with that in the other two scenarios.

Higher battery consumption in scenario three than in scenario one is because the more handover occurred. Based on the Equation (2), device energy consumption is based on the three different variables which are $d, \beta$ and T. In these three scenarios, values of $\beta$ are the same separately. The more number of handovers, the more time T wasted. From Table 2, the total numbers of handovers are 209, 171 and 257 in these three scenarios separately. Therefore, in Fig 6, decreased and increased values of energy consumption are proportional to scenario one. The more number of handovers generates the more battery consumption.

Table 2. Total Number of Handovers in each scenario

\begin{tabular}{|c|c|c|c|}
\hline & $\begin{array}{c}\text { Scenario } \\
\text { One }\end{array}$ & $\begin{array}{c}\text { Scenario } \\
\text { Two }\end{array}$ & $\begin{array}{c}\text { Scenario } \\
\text { Three }\end{array}$ \\
\hline $\begin{array}{c}\text { Total number of } \\
\text { Handover }\end{array}$ & 209 & 172 & 257 \\
\hline
\end{tabular}

Number of handover over the course of simulation are demonstrated in Fig 7, 8, and 9. It can be seen that average number of handovers in scenario two (Fig 8) is lower than those in scenario one (Fig 7). Reduced number of handovers of UEs can decrease values of battery consumption and confirms the results presented in Fig 6 . Higher number of handover can be observed in Fig 9 that correspond to the higher battery consumption of scenario three in Figure 6.

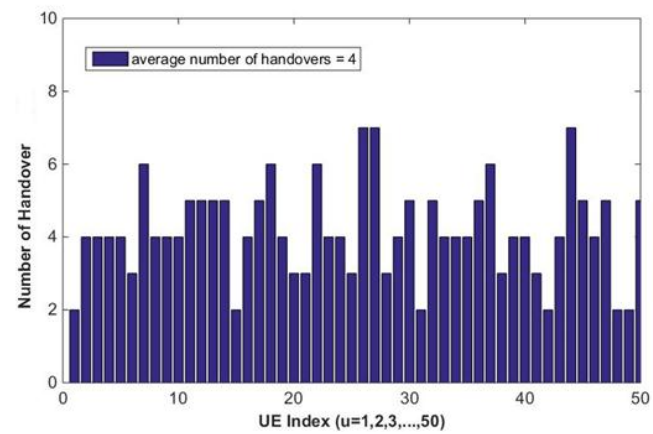

Figure 7. Number of Handovers in Scenario one (QoS-based)

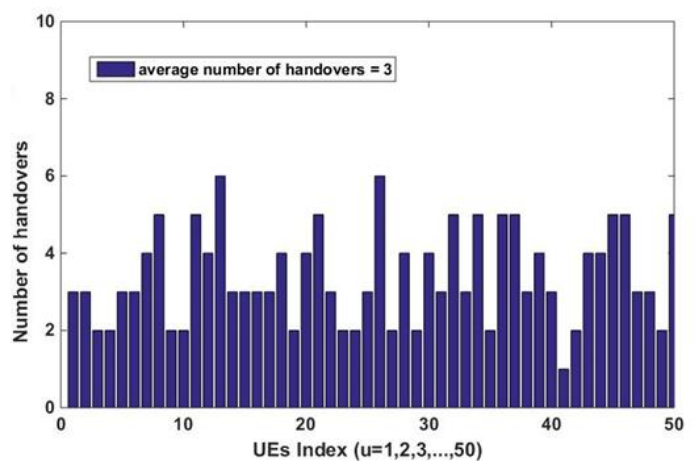

Figure 8. Number of Handovers in Scenario two (Learning-based) 


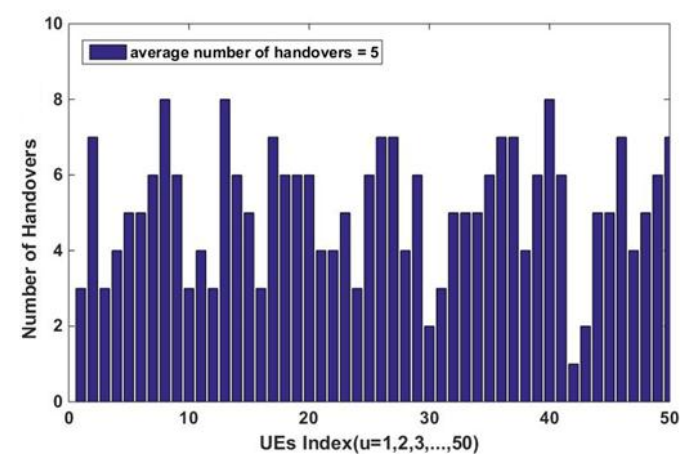

Figure 9. Number of Handovers in Scenario 3 (QFM-based)

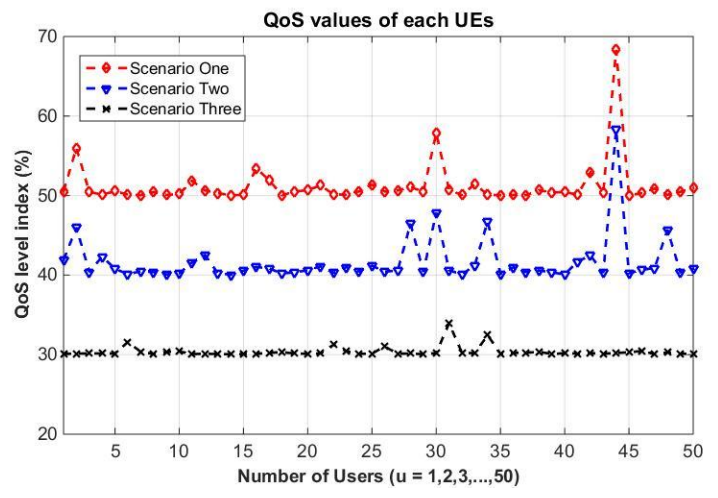

Figure 10. QoS Utility based on Equation (3)

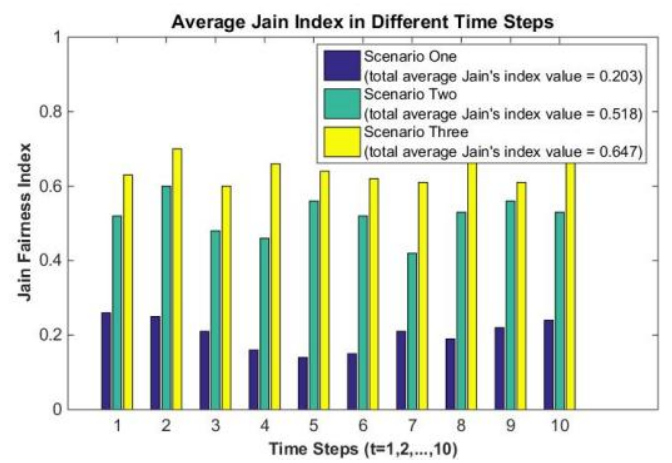

Figure 11. Average Jain Index in Vs simulation time steps

Furthermore, Fig 10 shows the QoS utility value (as Equation (3)). It can be seen that the QoS utility is lowest in scenario three and highest in scenario one. That is because, the aim of scenario one is enabling UEs to connect with optimal APs which can provide maximum value of QoS. Based on Equation (3), though the value of energy consumption are higher in scenario one, weight value of it is much smaller compared with throughput which is the main affect factor for QoS level. Therefore, QoS level of scenario two is lower than that in scenario one, even though the energy consumption has been reduced to a large extent. In scenario three, our proposed QFM mechanism implements fairness traffic mechanism which will reduce values of QoS for UEs at the same time. After UEs select APs by considering their selfish requirements, our cloud central controller reassign traffic resource to UEs with respect to fairness.

Finally, Jain's fairness index is plotted in Fig 11. As expected, scenario three has the highest fairness index and scenario one has the lowest fairness index. Based on fairness Equation (5), the value of $J(X)$ is in the field of $(0,1)$ and the higher the better. Higher $\mathrm{J}(\mathrm{X})$ value will be generated when values of $\left(\sum_{j=1}^{U} x_{j}\right)^{2}$ and $\left(U * \sum_{j=1}^{U} x_{j}^{2}\right)$ are quite similar. In scenario three, fairness problem (P2) has been solved by our proposed QFM mechanism. Network resource has been allocated efficiently while minimum requirements of UEs have been satisfied as well. In scenario one, each UEs purchases higher throughput and connects with the AP which can provide the highest resource at the same time. Therefore, the value of throughput will be quite different between each UEs and it will generate lowest fairness index in scenario one. 


\section{Conclusions}

In this paper, we presented a novel approach of traffic management in heterogeneous networks, which is QoS and Fairness Maximization (QFM) mechanism. With the rapid increasing number of mobile devices, their throughput demand and longer battery lifetime requirements, maximizing their QoS levels will be the significant part in the next generation networks. Meanwhile, how to allocate traffic resource in a fairness way is another important issue for us to consider. Our proposed QFM mechanism is composed by two parts which are fully distributed QoS maximization mechanism at UE side and centralized fairness traffic management mechanism at controller side. These two parts are implemented by UEs and cloud central controller separately with the whole view of the system. Based on analysis results, we can find that our proposed fairness problem have been solved. Resources of the network have been fairly allocated and the fairness index has been maximized as well.

\section{REFERENCES}

[1] H. Elshaer, F. Boccardi, M. Dohler, and R. Irmer, Downlink and uplink decoupling: A disruptive architectural design for $5 g$ networks. IEEE Global Communications Conference (GLOBECOM), pp. 1798-1803, December 2014.

[2] R. Guerzoni, R. Trivisonno, and D. Soldani, Sdn-based architecture and procedures for $5 g$ networks. 1st International Conference on 5G for Ubiquitous Connectivity (5GU), pp. 209-214, November 2014.

[3] T. Mahmoodi and S. Seetharaman, Traffic jam: Handling the increasing volume of mobile data traffic. IEEE Vehicular Technology Magazine, vol.9, pp. 56-62, September 2014.

[4] P. Ameigeiras, J. Ramos-Munoz, L. Schumacher, J. Prados-Garzon, J. Navarro-Ortiz, and J. Lopez-Soler, Link-level access cloud architecture design based on sdn for $5 g$ networks. IEEE Network, vol. 29, pp. 2431, March 2015.

[5] Cisco visual networking index: Global mobile data traffic forecast update 20142019. Cisco White paper, February 2015.

[6] D. Tuncer, M. Charalambides, S. Clayman, and G. Pavlou, Adaptive resource management and control in software defined networks. IEEE Transactions on Network and Service Management, vol. 12, pp. 18-33, March 2015.

[7] M. Jiang, Device-controlled traffic steering in mobile networks. 9th International Conference on Next Generation Mobile Applications, Services and Technologies, September 2015.

[8] Jain, R., D. Chiu, and W. Hawe. "A Quantitative Measure Of Fairness And Discrimination For Resource Allocation In Shared Computer Systems." arXiv preprint cs/9809099 (1998).

[9] Y. Zaki, T. Weerawardane, S. Hauth, E. Wallmeier, and C. Gorg, Intelligent traffic enforcement for Ite backhaul. IEEE International Symposium on Personal Indoor and Mobile Radio Communications (PIMRC), pp. 3077-3082, September 2013.

[10] C-RAN: The road Twoards Green RAN. China Mobile White paper, October 2011. 
Menglan Jiang and Toktam Mahmoodi; Traffic Management in 5G Mobile Networks: Selfish Users and Fair Network, Transactions on Networks and Communications, Volume 4 No. 1, February (2016); pp: 1-15

[11] F. Boccardi, R. Heath, A. Lozano, T. Marzetta, and P. Popovski, Five disruptive technology directions for $5 g$. IEEE Communications Magazine, vol. 52, pp. 74-80, February 2014.

[12] Q.-T. Nguyen-Vuong, N. Agoulmine, E. Cherkaoui, and L. Toni, Multicriteria optimization of access selection to improve the quality of experience in heterogeneous wireless access networks. IEEE Transactions on Vehicular Technology, vol. 62, pp. 1785-1800, May 2013.

[13] M. Louta and P. Bellavista, Bringing always-best connectivity vision a step closer: Challenges and perspectives. IEEE Communications Magazine, vol. 51, pp. 158-166, February 2013.

[14] P. Muoz, D. Laselva, R. Barco, and P. Mogensen, Dynamic traffic steering based on fuzzy q-learning approach in a multi-rat multi-layer wireless network. Computer Networks, vol. 71, pp. 100 - 116, 2014.

[15] K.L. A. Yau, P. Komisarczuk, and P. D. Teal, Reinforcement learning for context awareness and intelligence in wireless networks: Review, new features and open issues, Journal of Network and Computer Applications, vol. 35, no. 1, pp. 253 - 267, January 2012.

[16] Y. Kiran, T. Venkatesh, and C. Murthy, Reinforcement learning based path selection and wavelength selection in optical burst switched networks, International Conference on Broadband Communications, Networks and Systems (BROADNETS), October 2006.

[17] P. Munoz, R. Barco, D. Laselva, and P. Mogensen, Mobility-based strategies for traffic steering in heterogeneous networks. IEEE Communications Magazine, vol. 51, pp. 54-62, May 2013.

[18] T. Mahmoodi, V. Friderikos, O. Holland, and A. Hamid Aghvami, Cross-Layer Optimization to Maximize Fairness Among TCP Flows of Different TCP Flavors, IEEE Global Communications Conference (GLOBECOM), December 2008.

[19] T. Mahmoodi, V. Friderikos, O. Holland, and A. Hamid Aghvami, TCP-aware resource allocation in OFDMA based wireless networks, IEEE International Workshop on Cross Layer Design (IWCLD), June 2009.

[20] D. Cheng, J. Rao, C. Jiang, and X. Zhou, Elastic power-aware resource provisioning of heterogeneous workloads in self-sustainable datacenters. IEEE Transactions on Computers, vol. PP, no. 99, May 2015.

[21] C. Guo, M. Sheng, X. Wang, and Y. Zhang, Throughput maximization with short-term and long-term jain's index constraints in downlink ofdma systems. IEEE Transactions on Communications, vol. 62, pp. 15031517, May 2014.

[22] Access network discovery and selection function (ANDSF) management object (MO). 3GPP TS 24.312, September 2014.

[23] D. Lopez-Perez, I. Guvenc, G. de la Roche, M. Kountouris, T. Quek, and J. Zhang, Enhanced intercell interference coordination challenges in heterogeneous networks. IEEE Wireless Communications, vol. 18, pp. 22-30, June 2011.

[24] S. Alavi, C. Zhou, and W. W. Gen, Efficient and fair resource allocation scheme for ofdma networks based on auction game. IEEE Vehicular Technology Conference (VTC) Fall, September 2012. 
[25] J. Li, J. Xiao, J.-K. Hong, and R. Boutaba, Application-centric wi-fi energy management on smart phone. Asia-Pacific Network Operations and Management Symposium (APNOMS), September 2012.

[26] Y. Bai, J. Zhou, and L. Chen, Hybrid spectrum usage for overlaying Ite macrocell and femtocell. IEEE Global Communications Conference (GLOBECOM), November 2009.

[27] C. Ma and Y. Yang, A battery-aware scheme for routing in wireless ad hoc networks. IEEE Transactions on Vehicular Technology, vol. 60, pp. 3919-3932, October 2011.

[28] J. Cho, Y. Woo, S. Kim, and E. Seo, A battery lifetime guarantee scheme for selective applications in smart mobile devices. IEEE Transactions on Consumer Electronics, vol. 60, pp. 155-163, February 2014. 